\title{
遵从现行化学需氧量标准导致污水处理过程中 产生过量温室气体排放
}

\author{
吕宗青 ${ }^{1}$, 单晓雨 ${ }^{2}$, 肖喜林 ${ }^{1}$, 蔡阮鸿 $^{1}$, 张瑶 $^{1 \dagger}$, 焦念志 ${ }^{1 *}$
}

1. 厦门大学近海海洋环境科学国家重点实验室, 海洋与地球学院, 微型生物与地球圈层研究所, 厦门 361101 ;

2. 清华大学环境学院, 北京 100084

* 通讯作者, E-mail: jiao@xmu.edu.cn

†通讯作者, E-mail: yaozhang@xmu.edu.cn

收稿日期: 2021-05-20; 收修改稿日期: 2021-08-12; 接受日期: 2021-08-30; 网络版发表日期: 2021-11-16

国家自然科学基金委基础科学中心(筹)项目、国家重点研发计划项目(编号: 2016YFA0601400)和国家自然科学基金项目(批准号: 91751207 、 41861144018)资助

\begin{abstract}
摘要化学需氧量(COD)被广泛用作污水处理厂的有机污染指标. 污水处理过程中去除大量有机物以满足COD 环境标准, 该过程会伴随大量温室气体的释放, 例如甲烷. 然而, COD指标涵盖了大量不仅不是污染, 且可能是潜 在碳汇的难降解有机物. 本文收集并分析了来自全球86座市政污水处理厂的COD数据, 应用政府间气候变化专门 委员会发布的COD产甲烷模型来估算中国市政污水处理系统处理惰性有机物所造成的甲烷排放量. 结果表明, 污 水处理过程去除的有机物中平均有 $55 \%$ 的有机碳来自惰性有机物. 这部分惰性碳是本应保留的碳汇, 处理这部分 有机碳反而造成包括甲烷在内的温室气体排放. 以2018年为例, 中国市政污水处理系统去除惰性有机物所释放的 甲烷量可能高达 3822 万吨二氧化碳当量, 这与中国湿地每年所能固定的二氧化碳量相当. 这表明COD作为有机污 染的指标是不恰当的, 该指标需要修正. 事实上, 将无毒害的惰性有机物保留在环境中既可贡献碳汇, 又可降低污 水处理的能耗, 减少排放, 对于碳中和有重要意义.
\end{abstract}

关键词化学需氧量, 污水处理, 惰性溶解有机物, 温室气体排放

\section{1 引言}

自工业革命以来，温室气体排放成为气候变化的 主要驱动力(Hansen和Lacis，1990；Lashof和Ahuja, 1990; Montzka等, 2011), 其中污水处理厂是造成温室 气体排放的重要来源之一(Koutsou等, 2018; Nguyen 等，2019). 环境保护部门制定了严格的水质标准(Un-
ion和Parliament, 2013), 为了满足环境标准, 污水处理 过程须去除大量有机物和营养物质(Ra等, 2000; Zhang 等, 2020). 然而, 这些污水处理过程产生甲烷 $\left(\mathrm{CH}_{4}\right)$ 、 二氧化碳 $\left(\mathrm{CO}_{2}\right)$ 和一氧化二氮 $\left(\mathrm{N}_{2} \mathrm{O}\right)$ 等温室气体，排放 到大气中贡献于气候变暖(Gupta和Singh, 2012; Poulsen和Hansen, 2009).

其中 $\mathrm{CO}_{2}$ 的直接排放来自于生物处理过程中微生 
物对有机物的降解作用(Bao等, 2015). $\mathrm{CH}_{4}$ 则是来自于 有机物厌氧消化过程(anaerobic digestion)中的水解、 产乙酸及产甲烷作用(Ziemiński和Frąc, 2012). 污水处 理中最常用的生物处理技术(例如，厌氧/缺氧/好氧工 艺和序列间歇式活性污泥法)都会产生大量 $\mathrm{CH}_{4}$ 排放 (Bao等, 2016; Nguyen等, 2019). 先前的实验还表明, 污 水处理过程中包括好氧处理和厌氧处理在内的所有处 理环节都可以释放 $\mathrm{CH}_{4}$ (Wang等, 2011; Liu等, 2014). 全 球大气中的 $\mathrm{CH}_{4}$ 浓度已从工业时代之前的约 $715 \mathrm{ppb}$ (parts per billion, $1 \mathrm{ppb}=1 \mathrm{~nL} \mathrm{~L} \mathrm{~L}^{-1}$ )增加到2020年的 $1872 \mathrm{ppb}$ (https://www.esrl.noaa.gov). 在百年时间尺度 上, $\mathrm{CH}_{4}$ 的全球增温潜势 (global warming potential, GWP) 是 $\mathrm{CO}_{2}$ 的 28 倍(IPCC，2014), 必须引起足够重视. 而污水处理厂可贡献全球 $\mathrm{CH}_{4}$ 总排放量的 $5 \%$ (Chai等, 2015). $\mathrm{N}_{2} \mathrm{O}$ 的GWP远高于 $\mathrm{CH}_{4}$, 是 $\mathrm{CO}_{2}$ 的 265 倍(IPCC, 2014). $\mathrm{N}_{2} \mathrm{O}$ 通常是污水处理中反硝化过程的中间产物, 来自于有机物对硝酸盐或亚硝酸盐的还原(Hanaki等, 1992; Wunderlin等, 2012). 据估计, 到2030年, 全球污水 处理厂的 $\mathrm{CH}_{4}$ 和 $\mathrm{N}_{2} \mathrm{O}$ 排放量将分别超过 6 亿吨和 1 亿吨 $\mathrm{CO}_{2}$ 当量 $\left(\mathrm{CO}_{2} \mathrm{e}\right)$ (Ragnauth等, 2015). 鉴于污水处理是人 类主动行为, 相对于其他不可控的自然环境, 污水处理 厂是人为可控环节. 全面认识污水处理过程中的科学 问题、制定恰当的水质标准和环境政策，对于落实碳 中和战略具有尤其重要的意义.

污水处理过程中有机物的浓度普遍采用化学需氧 量(chemical oxygen demand, COD)指标. 目前国际通用 的 COD处理方法不仅氧化了生物可降解的有机碳、 而且氧化了生物难降解的有机碳组分(Dulekgurgen等, 2006). 另一个指标是生化需氧量(biochemical oxygen demand, BOD), 它表征了有机物中易于被生物降解的 组分(Samudro和Mangkoedihardjo, 2010; Jouanneau等, 2014). 为了达到污水排放的水质标准，污水处理工艺 追求最大程度地降低COD和BOD(Cotillas等，2018; Freeman等, 2018; Verma和Suthar, 2018). 生物处理是 污水处理中降低COD与BOD的主要手段, 主要依靠于 微生物的分解代谢和合成代谢(包括好氧消化和厌氧 消化过程(de Souza等，2010；Mittal，2011; Zhang等, 2020). 其中，处理惰性有机物更依赖于厌氧消化(Barker等, 1999), 而厌氧消化过程高达 $80 \%$ 的COD有机物 可被转化为 $\mathrm{CH}_{4}$ (Foley等, 2011).

然而, $\mathrm{COD}$ 氧化的有机物中有一类重要组分是惰 性溶解有机碳(recalcitrant dissolved organic matter, RDOM)(Jiao等, 2021), 占自然水体环境总溶解有机物 的相当一部分份额，是重要的水体碳汇(Jiao等，2010; Hansell, 2013). RDOM广泛存在于污水处理厂的入水 和出水中(Archibald等, 1998; Bockhorn等, 2005; Jin等, 2011; Lu等, 2018). 例如, 在瑞士的一个典型污水处理 厂中, RDOM对总有机物的相对贡献高达 $91 \%$ (Kappeler和Gujer, 1992). 污水处理的厌氧消化过程对RDOM 的去除不仅增加能耗、而且伴随大量的 $\mathrm{CH}_{4}$ 释放 (Chelliapan等, 2012).

$\mathrm{BOD}$ 反映了水体中生物降解有机物所消耗的氧 气量(Reynolds, 2002). 因此, COD和BOD之间的差值 可以用作指征天然水体中的惰性有机物(Jiao等, 2021), 本文定义其为惰性 COD(recalcitrant COD, rCOD). 厌氧 消化过程能够降解被归类为 $\mathrm{rCOD}$ 的惰性有机物. 不同 于自然水体的生物降解，该过程通常需要满足某些极 端条件，例如极低的 $\mathrm{pH}$ 、高温和高缓冲能力等(Ward 等, 2008). 为了计算 $\mathrm{rCOD}$ 去除过程中产生的 $\mathrm{CH}_{4}$ 总量, 本文收集了世界各地市政污水处理厂进出污水的 $\mathrm{COD}$ 与 BOD数据, 估算出 $\mathrm{CCOD}$ 去除对总 $\mathrm{COD}$ 去除量 的相对贡献. 本文还估算了中国2017年和2018年市政 污水处理中去除COD所产生的 $\mathrm{CH}_{4}$ 排放量. 通过调研 水质监测指标导致的温室气体排放, 本文为污水处理 行业实现“碳中和”提供了新的视角.

\section{2 材料与方法}

\section{1 全球污水处理厂的数据采集}

全球水生微生物联盟(http://gwmc.ou.edu)于 2014 年成立，并提供了来自世界各地市政污水处理厂的样 本数据(Wu等, 2019). 通过该网站, 我们获取了来自全 球100座市政污水处理厂包括经纬度、实际流量、进 水 $\mathrm{BOD}\left(\mathrm{BOD}_{\mathrm{in}}\right)$ 、出水 $\mathrm{BOD}\left(\mathrm{BOD}_{\mathrm{ef}}\right)$ 、进水 $\mathrm{COD}\left(\mathrm{COD}_{\mathrm{in}}\right)$ 和出水 $\mathrm{COD}\left(\mathrm{COD}_{\mathrm{ef}}\right)$ 在内的数据集, 然后 剔除了 14 组BOD值显著高于COD值的存疑数据. 最终 获得了来自五大洲的 86 座污水处理厂的数据，这些污 水处理厂的地理分布如图1所示, 具体数据记录于网 络版附表S1中(http://earthen.scichina.com).

\section{2 污水处理厂rCOD去除率的估算}

$\mathrm{BOD}$ 通常分为 5 日 $\mathrm{BOD}\left(\mathrm{BOD}_{5}\right) 、 20$ 日 BOD 


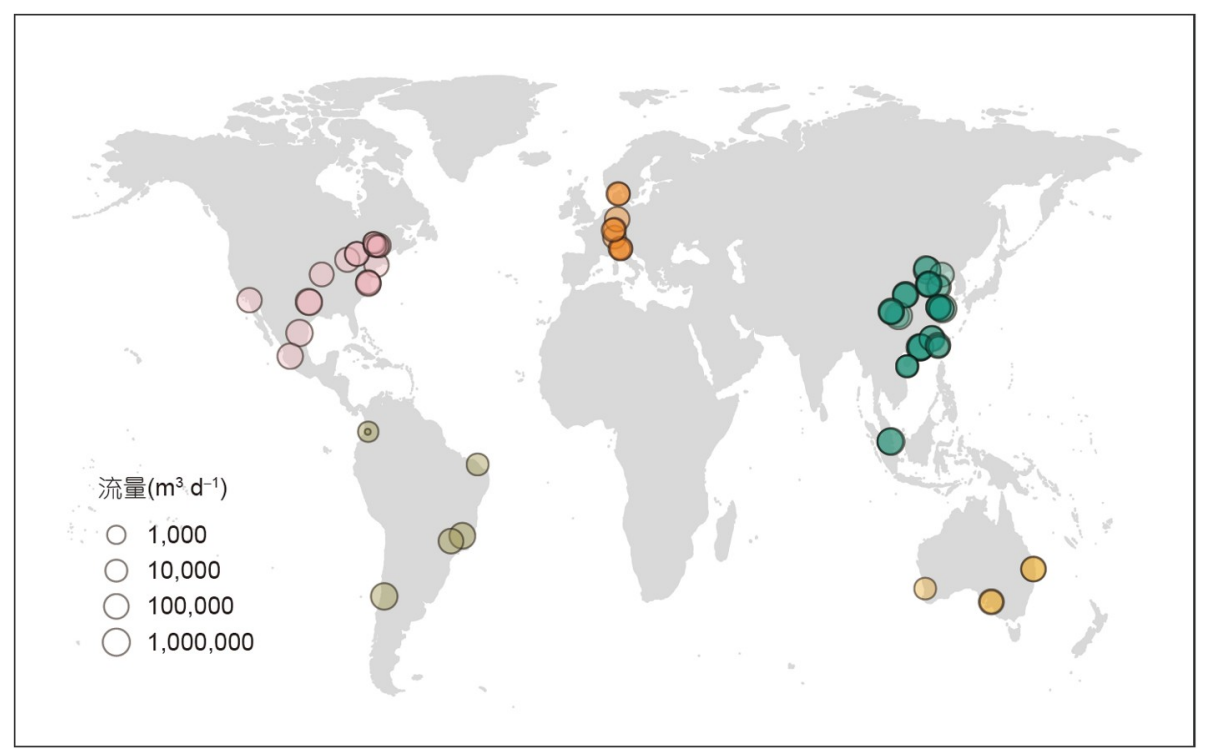

图 1 全球86座市政污水处理厂的地理分布

每个圆代表一座污水处理厂, 其流量与圆的面积成正比. 样本遍布五大洲, 即亚洲 $(n=46)$ 、欧洲 $(n=9)$ 、北美洲 $(n=20)$ 、南美洲 $(n=6)$ 、大洋洲 $(n=5)$. 地图改编自Wu等(2019)

$\left(\mathrm{BOD}_{20}\right)$ 和最终 $\mathrm{BOD}\left(\mathrm{BOD}_{\mathrm{u}}\right)$ (Bernardo等, 2011). 我们获 取的数据集中仅包含 $\mathrm{BOD}_{5}$, 根据经验值, $\mathrm{BOD}_{5}$ 约等于 有机物完全氧化分解所需的氧气总量的 $66 \%$, 而 $\mathrm{BOD}_{\mathrm{u}}$ 则可以看作是微生物在相当长的一段时间内消耗的氧 气总量(Makinia等, 2002; Huo等, 2006). 因此, 我们用 数据集中的 $\mathrm{BOD}_{5}$ (见网络版附表 $\mathrm{S} 1$ ) 除以 $66 \%$ 来估算污 水处理厂的 $\mathrm{BOD}_{\mathrm{u}}$. 将 $\mathrm{COD}$ 与 $\mathrm{BOD}_{5}$ 之差值定义为 $\mathrm{rCOD}_{5}, \mathrm{COD}$ 与 $\mathrm{BOD}_{\mathrm{u}}$ 之差值定义为 $\mathrm{rCOD}_{\mathrm{u}} \cdot \mathrm{rCOD}_{\mathrm{u}}$ 代表 更难降解的有机物. COD去除量可以通过进出污水的 $\mathrm{COD}$ 之差来计算. $\mathrm{rCOD}$ 去除量的计算公式如下:

$$
\begin{aligned}
\mathrm{rCOD}_{\text {Removal }} & =\mathrm{rCOD}_{\text {in }}-\mathrm{rCOD}_{\text {ef }} \\
& =\left(\mathrm{COD}_{\text {in }}-\mathrm{BOD}_{\text {in }}\right)-\left(\mathrm{COD}_{\mathrm{ef}}-\mathrm{BOD}_{\mathrm{ef}}\right),
\end{aligned}
$$

式中, $\mathrm{rCOD}_{\text {in }}$ 代表流入污水的 $\mathrm{rCOD}, \mathrm{rCOD}_{\mathrm{ef}}$ 代表流出 污水的rCOD.

然后, 对进水、出水和去除过程中的 $\mathrm{rCOD}$ 与 $\mathrm{COD}$ 进行了基于交叉验证的无截距最小二乘法回归. 每个 回归交叉验证999次, 其中一半 $(n=43)$ 样本被用作训练 集, 另一半 $(n=43)$ 样本被用作验证集. 交叉验证的 $R^{2}$ 计 算公式为

$$
R^{2}=1-\frac{\mathrm{RSS}}{\mathrm{TSS}}=1-\frac{\sum_{i=1}^{n}\left(y_{i}-\widehat{y}_{i}\right)^{2}}{\sum_{i=1}^{n}\left(y_{i}-\bar{y}\right)^{2}},
$$

式中, RSS 是残差平方和, TSS 是总平方和, $\widehat{y}_{i}$ 是验证集 中第 $i$ 个样本的拟合值, $y_{i}$ 是验证集中第 $i$ 个样本的观测 值, $\bar{y}$ 是验证集中所有样本观测值的平均值.

\subsection{COD转换 $\mathrm{CH}_{4}$ 的估算模型}

为了估算中国污水处理厂去除 $\mathrm{rCOD}$ 所产生的 $\mathrm{CH}_{4}$ 排放量, 以公式(1)和(2)估算得到的全球86座污水 处理厂的 $\mathrm{rCOD}$ 去除量在 $\mathrm{COD}$ 去除量中的平均比率, 代入公式(3)来估算中国市政污水处理中rCOD的年去 除量 $(\Delta \mathrm{rCOD})$.

$\Delta \mathrm{rCOD}=\Delta \mathrm{COD} \times$ 平均去除率,

其中 $\triangle \mathrm{COD}$ 是中国市政污水处理厂去除的COD总量, 2017 年和 2018 年的数据分别为 1180 和 1241 万吨 (http:// www.mee.gov.cn).

通过公式(4)(IPCC，2006)估算中国市政污水处理 厂去除的 $\Delta \mathrm{rCOD}$ 导致的最大 $\mathrm{CH}_{4}$ 排放量 $\left(\mathrm{ECH}_{4}\right)$ :

$\mathrm{ECH}_{4}=\Delta \mathrm{rCOD} \times \mathrm{EF} \times \mathrm{MCF}-\mathrm{R}$,

式中, $\mathrm{EF}$ 是最大甲烷产生/排放因子(Emission factor, $\left.\mathrm{EF}=0.25 \mathrm{~kg} \mathrm{CH} \mathrm{CH}_{4} / \mathrm{kg} \mathrm{COD}\right), \mathrm{MCF}$ 是不同污水处理过程 的甲烷修正系数 (methane correction factor, MCF如网 络版附表 $\mathrm{S} 2$ 所示), $\mathrm{R}$ 代表 $\mathrm{CH}_{4}$ 回收后用作燃料或转移 
到污水处理厂之外的量. 本研究只考虑 $\mathrm{CH}_{4}$ 的最大产 量, 不考虑后续 $\mathrm{CH}_{4}$ 回收等过程.

\section{3 结果与讨论}

\subsection{COD中的 $\mathrm{COD}$ 占比}

图2显示了全球86座污水处理厂流入和流出污水 的BOD和rCOD浓度. 我们发现, BOD被去除的同时总 是伴随着 $\mathrm{rCOD}$ 的去除. $\mathrm{BOD}$ 的减少是由微生物的分解 代谢和合成代谢造成的, 而伴随的 $\mathrm{rCOD}$ 的减少可能是 因为污泥消化或为达到严格的 COD排放标准所采取 的后续污染物去除手段造成的. 另外, 一小部分无机离 子也可以贡献于COD和BOD的值, 例如, 亚硝酸根、 硫酸根和亚铁离子可贡献于COD值(Kylefors等, 2003;
Samudro和Mangkoedihardjo, 2010)), 硫酸盐和亚铁离 子可贡献于BOD值(Hudson等, 2008; Yu等, 2016).

为了定量表征 $\mathrm{COD}$ 和 $\mathrm{rCOD}$ 之间的关系, 我们做了 $\mathrm{rCOD}\left(\mathrm{rCOD}_{5}\right.$ 和 $\mathrm{rCOD}_{\mathrm{u}}$ )与 $\mathrm{COD}$ 的无截距线性回归(图 3). 我们发现在进水中(图3a) $\mathrm{rCOD}_{5}$ 贡献了 $\mathrm{COD}$ 总量的 $56 \%\left(R^{2}=0.95, p<10^{-16}\right)$, 表明 $\mathrm{COD}$ 的大部分都是 $\mathrm{rCOD}_{5}$. 在流出污水中 (图 $3 \mathrm{c}$ ) $\mathrm{rCOD}_{5}$ 贡献了 $\mathrm{COD}$ 总量的 $74 \%$ $\left(R^{2}=0.78, p<10^{-16}\right)$, 表明污水处理后有机物的可生化性 降低. 图 $3 \mathrm{~b}$ 中, $\mathrm{rCOD}_{5}$ 的去除量是流入与流出污水 $\mathrm{rCOD}_{5}$ 之间的差值, 它对 $\mathrm{COD}$ 去除量的总体贡献为 $55 \%\left(R^{2}=0.93, p<10^{-16}\right)$. 这意味着每去除1单位的 $\mathrm{COD}$, 就有 0.55 单位的 $\mathrm{rCOD}_{5}$ 被去除. 估算出的 $\mathrm{rCOD}_{\mathrm{u}}$ 占流入 污水 $\mathrm{COD}$ 的 $33 \%\left(R^{2}=0.75, p<10^{-16}\right)$ (图3d), 占流出污水 $\mathrm{COD}$ 的 $61 \%\left(R^{2}=0.67, p<10^{-16}\right)$ (图3f), 以及COD去除量
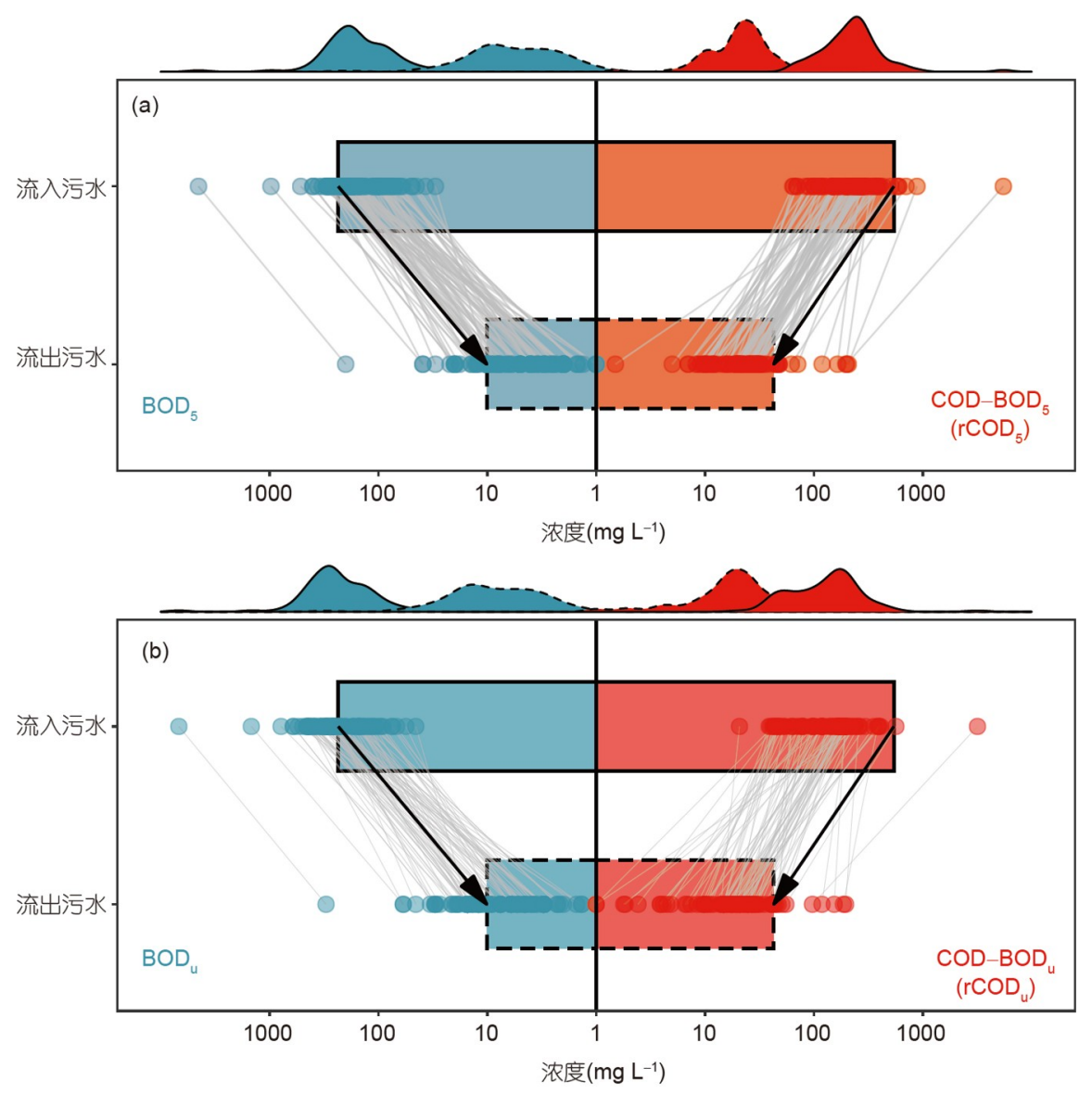

图 2 流入和流出污水的BOD与rCOD

污水处理去除的 $\mathrm{COD}$ 由 $\mathrm{BOD}$ (蓝色) 和 $\mathrm{rCOD}$ (红色)组成, $\mathrm{rCOD}$ 定义为 $\mathrm{COD}-\mathrm{BOD}$. (a)和(b)分别显示了 $\mathrm{BOD}_{5}$ 和 $\mathrm{rCOD} \mathrm{D}_{5}$ 以及 $\mathrm{BOD}_{\mathrm{u}}$ 和 $\mathrm{rCOD}_{\mathrm{u}}$ 的浓 度变化. 同一污水处理厂流入和流出污水的数据点由灰线连接. 条形图显示了污水处理厂流入污水(实线)和流出污水(虚线)BOD和 $\mathrm{rCOD}$ 的平 均浓度. 顶部曲线显示了流入和流出污水BOD和rCOD的密度分布 

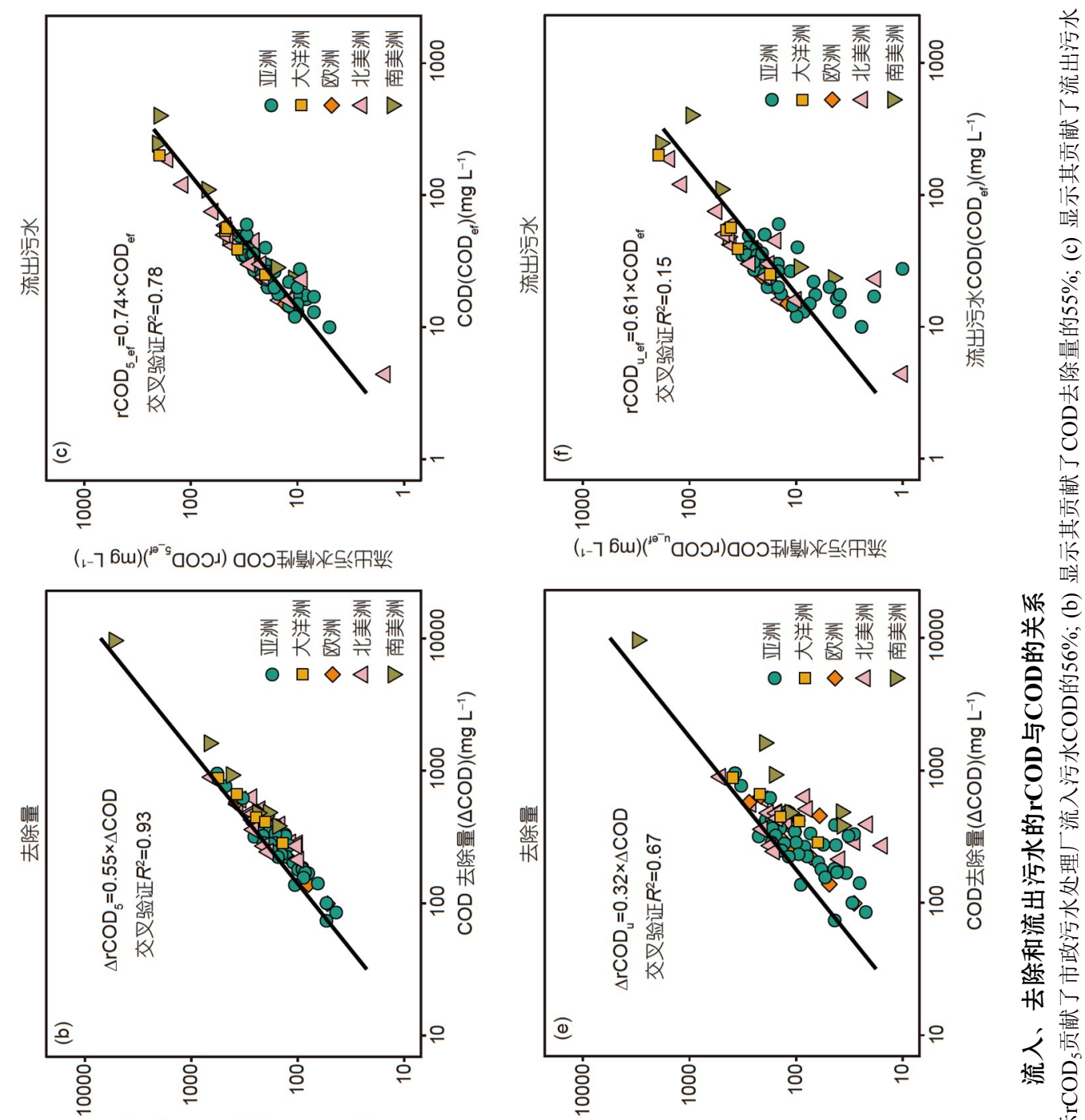

Whe

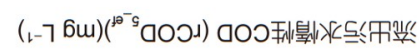

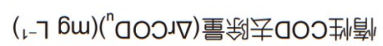
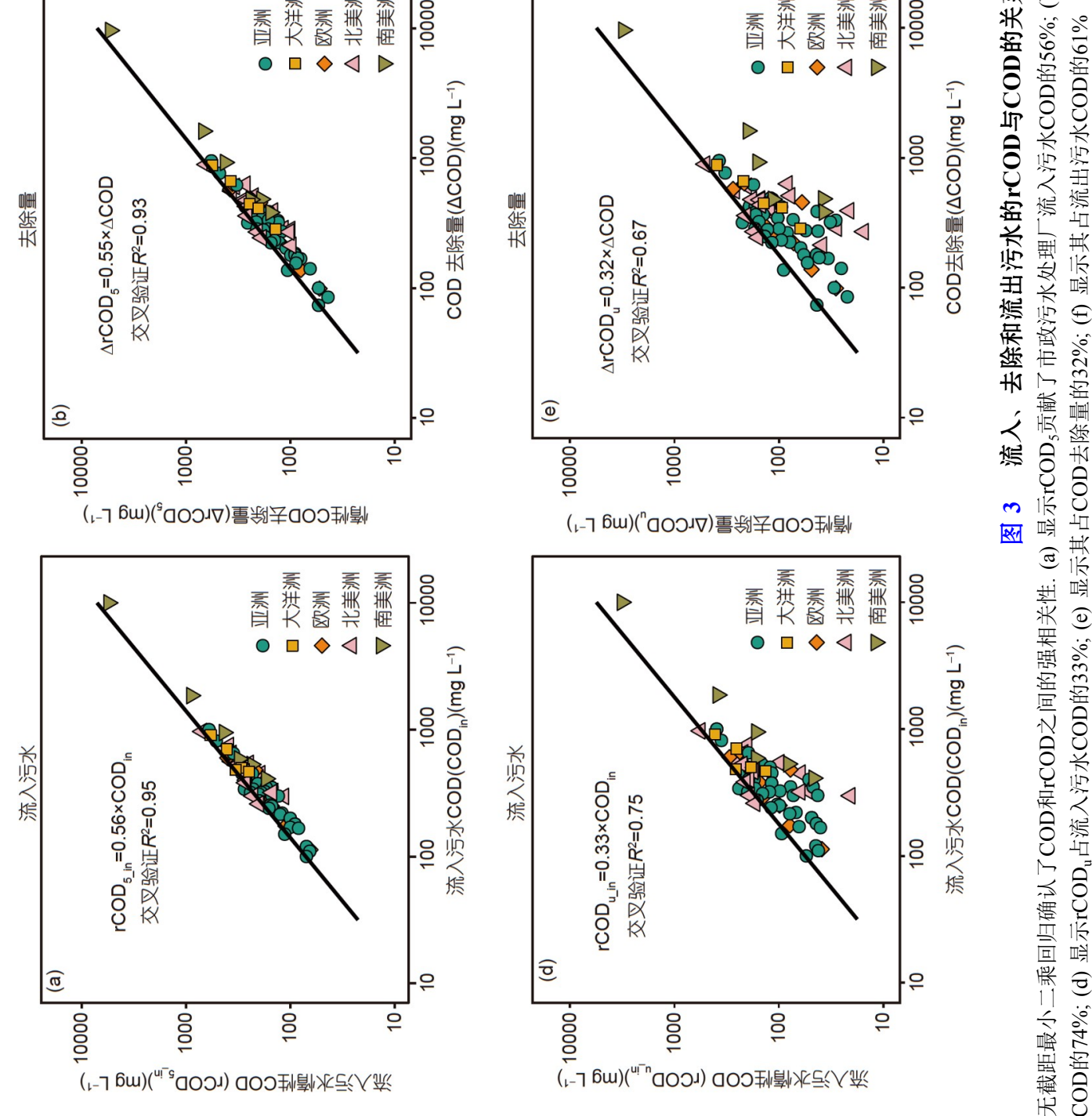
的 $32 \%\left(R^{2}=0.15, p<10^{-16}\right)$ (图3e). 这说明污水处理去除 的COD有机物中至少有 $32 \%$ 是惰性有机物. 将此回归 模型应用于中国的COD年去除量时，我们估算得出, 2017 年市政污水处理厂去除的 $\mathrm{rCOD}_{5}$ 和 $\mathrm{rCOD}_{\mathrm{u}}$ 的总量 分别为 649 万吨和 378 万吨， 2018 年分别为 683 万吨和 399万吨.

图3a也表明，当把流入污水的 $\mathrm{COD}$ 分为 $\mathrm{BOD}$ 和 $\mathrm{rCOD}$ 时, BOD占到COD总量的一半以下. 这表明污水 中大约一半的有机物在天然水环境中不能被好氧微生 物迅速降解. 这些惰性有机物主要由高分子量的腐殖 质和低分子量的微生物产物组成(Lu等, 2018). 腐殖质 和富里酸类化合物不仅大量存在于流入污水中，还存 在于污水处理过程中, 是污水好氧和厌氧生物处理的 主要副产物(Guo等, 2011). 据估算, 微生物产生的有 机物的COD测量值可以占到COD总量的 $2.2 \%$ (Ince等, 2000). 这些微生物产生的有机物可以抵抗生物降解, 并能够在天然水体中长期储存 (Jiao等, 2010). 然而, 在 污水处理过程中, 这类有机物在后续的反硝化和除磷 过程中会被进一步去除，这促进了温室气体的产生 (Lemaire等, 2006; Yan等, 2020). 不同于天然条件, 污 水处理过程通常在特定和严苛的条件下进行, 因此能 够去除RDOM(Ward等, 2008).

\section{2 由 $\Delta r C O D$ 转化的温室气体量}

厌氧消化在降解惰性有机物过程中起重要作用 (Wang等, 2012). 尽管大多数污水处理厂(尤其在中国) 不只使用厌氧处理(Fang等, 2016), 为了估算去除惰性 有机物而产生的最大温室气体排放潜力, 我们假设 $\mathrm{rCOD}$ 全部通过厌氧过程去除, 并且估算中国市政污水 处理厂的 $\mathrm{CH}_{4}$ 排放量时使用了 $\mathrm{CH}_{4}$ 修正因子的最大值 $(\mathrm{MCF}=0.8$; 网络版附表 $\mathrm{S} 2)$. 同时为了更简单的比较, $\mathrm{CH}_{4}$ 被转换为 $\mathrm{CO}_{2} \mathrm{e}(\mathrm{GWP}=28)$. 研究发现, 如果不考虑 $\mathrm{CH}_{4}$ 回收, 在2017年和 2018 年, 由 $\Delta \mathrm{rCOD}_{5}$ 转化为 $\mathrm{CH}_{4}$ $\left(\mathrm{CO}_{2} \mathrm{e}\right)$ 的最大潜力分别为 $130(3634)$ 和137(3822)万吨. 由 $\Delta \mathrm{rCOD}_{\mathrm{u}}$ 转化为 $\mathrm{CH}_{4}\left(\mathrm{CO}_{2} \mathrm{e}\right)$ 的最大潜力分别为 76 (2115)和79(2224)万吨.

正如前所属述, 因为厌氧处理(主要过程)不是去 除 $\mathrm{rCOD}$ 的唯一过程, 还有一小部分 $\mathrm{rCOD}$ 是通过强化 的后处理过程去除的，例如好氧抛光、臭氧化、电凝 和电子束辐照等技术(Kallas和Munter, 1994; Barker等, 1999; Makwana和Ahammed, 2017; Deogaonkar等,
2019), 这些处理过程释放的 $\mathrm{CH}_{4}$ 较少，因此我们估算 所得的 $\mathrm{CH}_{4}$ 排放最大潜能可能被高估. 此外, 我们在估 算 $\mathrm{CH}_{4}$ 最大排放潜力时没有考虑 $\mathrm{CH}_{4}$ 的回收. 先前的研 究估算, 从 $\mathrm{CH}_{4}$ 中回收能量可以补偿污水处理过程中 消耗的能量的一半, 从而减少至少 $50 \%$ 的间接 $\mathrm{CO}_{2}$ 排 放(郝晓地等, 2014; Hao等, 2015; dos Santos等, 2016), 这有助于污水处理厂实现碳中和的目标.

为确保本文模型估算的可靠性, 将估算数据与中 国政府公布的官方数据进行了比较. 当将 $\mathrm{rCOD}_{5}$ 去除 率 $(55 \%)$ 应用于2014年中国污水处理行业相关数据时 (http://www.mee.gov.cn), $\mathrm{CH}_{4}$ 排放总量(217万吨)中有 149 万吨是由 $\Delta \mathrm{rCOD}_{5}$ 所贡献的, 这甚至高于我们所估 算的2018年 $\mathrm{CH}_{4}$ 排量的最大潜能估算值(137万吨). 这 也在一定程度上反映了中国污水处理行业在 $\mathrm{CH}_{4}$ 回收 利用作为能源方面还亟待发展.

表1显示，2018年去除rCOD导致的温室气体排放 量约为2224 3822万吨 $\mathrm{CO}_{2} \mathrm{e}$. 与能源、交通等碳密集 型行业相比, 污水处理减排成本低且效益明显. 因此 污水处理行业已被许多国家列为减排和实现“碳中和” 的优先行业(Mo和Zhang, 2012). 如果按照林业碳汇的 算法来计算, 每年去除 $\mathrm{rCOD}$ 所排放的 $\mathrm{CO}_{2}$ 量需要大约 250 400万公顷的植树造林才能抵消(李怒云，2007), 这将耗资310 530亿美元. 此外, 这些温室气体排放量 可以占到2014年中国湿地(重要的碳汇生态系统 (Mitsch等, 2013; Chen等, 2017))总固碳量(4454万吨)的 50 86\%(http://www.mee.gov.cn).

$\mathrm{N}_{2} \mathrm{O}$ 是COD去除的另一个重要产物, 其由于脱氮 不完全而产生, 被认为是污水脱氮过程中不可避免的 中间产物. 并且, 当加强限制 COD时会导致更多的 $\mathrm{N}_{2} \mathrm{O}$ 产生(Shannon等, 2008), 以往的研究发现, 碳短缺 环境(低COD/N比)会导致反硝化细菌利用自身碳源进 行反硝化(Wu等, 2009; 耿军军等, 2010), 这会导致亚 硝酸盐的积累, 然后产生更多的 $\mathrm{N}_{2} \mathrm{O}$, 从而加剧温室气 体的排放(Rodriguez-Garcia等, 2012).

\section{3 寻求污水处理效率与温室气体排放之间的平 衡}

未来的污水处理不仅要追求有机污染物的减少, 还要追求有机物去除效率和温室气体排量降低之间的 平衡. 经典的COD指标有其缺陷, 事实上, 高COD值并 不一定对应于较差的水质. 有许多水质较好的天然水 
表 12017 和 2018年中国市政污水处理去除rCOD所产生 的温室气体年排放潜力估算

\begin{tabular}{ccccc}
\hline \multirow{2}{*}{ 年份 } & \multicolumn{4}{c}{ 厌氧消化产生的温室气体排放量 ${ }^{\mathrm{a})}$ (万吨) } \\
\cline { 2 - 5 } & $\mathrm{ECH}_{4}{ }^{\mathrm{b}}{ }^{\mathrm{b}}$ & $\mathrm{CO}_{2} \mathrm{e}^{\mathrm{b}), \mathrm{c}}$ & $\mathrm{ECH}_{4}{ }^{\mathrm{d})}$ & $\mathrm{CO}_{2} \mathrm{e}^{\mathrm{c}), \mathrm{d})}$ \\
\hline 2017 & 130 & 3634 & 76 & 2115 \\
2018 & 137 & 3822 & 79 & 2224 \\
\hline
\end{tabular}

a) $\mathrm{CH}_{4}$ 回收未被考虑, b) 由估算的 $\Delta \mathrm{rCOD}_{5}$ 转化得来, c) $\mathrm{CH}_{4}$ 转 化为 $\left.\mathrm{CO}_{2} \mathrm{e}(\mathrm{GWP}=28), \mathrm{d}\right)$ 由估算的 $\Delta \mathrm{rCOD}_{\mathrm{u}}$ 转化得来

环境，由于富含RDOM而导致COD不达标(Aoki等, 2004; Räike等, 2012; Jiao等, 2021). 据报道, 造纸厂的 废水含有大量木质素相关的惰性有机物，这些物质可 以产生COD值，然而其不仅无毒，且与天然有机物质 非常相似(Archibald等，1998). 此外，高COD值也不一 定等于水体富营养化(Guo等, 2017).

随着人类社会的发展，水中溶解有机物的含量必 然会增加(Sepp等, 2018). 如果不加选择地去除溶解有 机物，大量的碳将被排放到大气中，进而加剧气候变 化. 随着污水处理技术的进步，越来越多的有机物将 被去除，以达到COD标准. 因此，如果不修改COD指 标，高COD去除率将伴随着更高的温室气体直接排放 量. 水处理行业应完善水质监测指标，如区分污水中 有机物的特性、可生物降解性及其在天然环境中的归 宿等，以及区分对人类和其他生物有害的无机离子和 有机物. 改进水质监测指标将有助于把无毒害的 $\mathrm{RDOM}$ 作为碳汇保存下来，从而以更经济的方式实现 污水处理行业的“碳中和”.

\section{4 结论}

污水处理行业是全球碳循环的重要组成环节，现 行的污水处理过程在降低有机碳的同时释放大量的 $\mathrm{CO}_{2} 、 \mathrm{CH}_{4}$ 和 $\mathrm{N}_{2} \mathrm{O}$, 对气候变化产生直接影响. 造成这 种现状的一个重要原因是环境指标导向. COD作为普 遍使用的水质指标，只考虑了有机污染，没有顾及温 室气体排放，这有悖于“碳中和”理念。本文通过世界 范围内 86 座污水处理厂的数据分析表明, 在目前污水 处理行业的污水排放COD标准下，本来可以作为水体 碳汇的RDOM被大量去除，并释放大量温室气体到大 气中. 2018 年中国市政污水处理厂的理论排放量最大 可高达 3822 万吨 $\mathrm{CO}_{2}$ 当量, 这几乎与中国湿地每年固
碳总量相当. 因此，污水处理行业应用的COD指标标 准需要修正. 未来需要做更多的工作来优化水质指标 及其标准，同时兼顾水质改善和温室气体减排，以利 于污水处理行业落实“碳中和”战略.

致谢 感谢宁大亮博士、张冰博士、周集中教授和杨云 锋教授在数据方面的支持与帮助.

\section{参考文献}

耿军军, 王亚宜, 张兆祥, 任中佳, 何维涛. 2010. 污水生物脱氮革新 工艺中强温室气体 $\mathrm{N}_{2} \mathrm{O}$ 的产生及微观机理. 环境科学学报, 30 : 1729-1738

郝晓地, 刘然彬, 胡沅胜. 2014. 污水处理厂“碳中和”评价方法创建 与案例分析. 中国给水排水, 30: 1-7

李怒云. 2007. 中国林业碳汇. 北京: 中国林业出版社. 200

Aoki S, Fuse Y, Yamada E. 2004. Determinations of humic substances and other dissolved organic matter and their effects on the increase of COD in Lake Biwa. Anal Sci, 20: 159-164

Archibald F, Roy-Arcand L, Méthot M, Valeanu L. 1998. Recalcitrant organic compounds (chemical oxygen demand sources) in biologically treated pulp and paper mill effluents: Their fate and environmental impact in receiving waters. Water Environ Res, 70: 1314-1326

Bao Z, Sun S, Sun D. 2015. Characteristics of direct $\mathrm{CO}_{2}$ emissions in four full-scale wastewater treatment plants. Desalin Water Treat, 54 : 1070-1079

Bao Z, Sun S, Sun D. 2016. Assessment of greenhouse gas emission from $\mathrm{A} / \mathrm{O}$ and $\mathrm{SBR}$ wastewater treatment plants in Beijing, China. Int Biodeter Biodegr, 108: 108-114

Barker D J, Mannucchi G A, Salvi S M L, Stuckey D C. 1999. Characterisation of soluble residual chemical oxygen demand (COD) in anaerobic wastewater treatment effluents. Water Res, 33: 2499-2510

Bernardo M, Santos A, Cantinho P, Minhalma M. 2011. Biodegradation rate constants in different $\mathrm{NF} / \mathrm{UF}$ fractions of cork processing wastewaters. Desalin Water Treat, 29: 264-270

Bockhorn H, Braun A M, Frimmel F H, Reimert R, Syldatk C. 2005. Das Engler-Bunte-Institut der Universität Karlsruhe (TH) in den Jahren 2003/2004. GWF, 146: 432-452

Chai C, Zhang D, Yu Y, Feng Y, Wong M. 2015. Carbon footprint analyses of mainstream wastewater treatment technologies under different sludge treatment scenarios in China. Water, 7: 918-938

Chelliapan S, Mahat S B, Din M, Yuzir A, Othman N. 2012. Anaerobic digestion of paper mill wastewater. Iran J Energy Environ, 3: 85-90 
Chen H, Popovich S, McEuen A, Briddell B. 2017. Carbon and nitrogen storage of a restored wetland at Illinois' Emiquon preserve: Potential for carbon sequestration. Hydrobiologia, 804: 139-150

Cotillas S, Llanos J, Cañizares P, Clematis D, Cerisola G, Rodrigo M A, Panizza M. 2018. Removal of Procion Red MX-5B dye from wastewater by conductive-diamond electrochemical oxidation. Electrochim Acta, 263: 1-7

Deogaonkar S C, Wakode P, Rawat K P. 2019. Electron beam irradiation post treatment for degradation of non biodegradable contaminants in textile wastewater. Radiat Phys Chem, 165: 108377

dos Santos I F S, Vieira N D B, Barros R M, Filho G L T, Soares D M, Alves L V. 2016. Economic and $\mathrm{CO}_{2}$ avoided emissions analysis of WWTP biogas recovery and its use in a small power plant in Brazil. Sust Energy Technol Assess, 17: 77-84

Dulekgurgen E, Doğruel S, Karahan O, Orhon D. 2006. Size distribution of wastewater COD fractions as an index for biodegradability. Water Res, 40: 273-282

Fang F, Qiao L L, Cao J S, Li Y, Xie W M, Sheng G P, Yu H Q. 2016. Quantitative evaluation of $\mathrm{A}^{2} \mathrm{O}$ and reversed $\mathrm{A}^{2} \mathrm{O}$ processes for biological municipal wastewater treatment using a projection pursuit method. Sep Purif Technol, 166: 164-170

Foley J, Yuan Z, Keller J, Senante E, Chandran K, Willis J, Shah A, van Loosdrecht M, van Voorthuizen E. 2011. $\mathrm{N}_{2} \mathrm{O}$ and $\mathrm{CH}_{4}$ emission from wastewater collection and treatment systems. Technical Report. Global Water Research Coalition

Freeman A I, Surridge B W J, Matthews M, Stewart M, Haygarth P M. 2018. New approaches to enhance pollutant removal in artificially aerated wastewater treatment systems. Sci Total Environ, 627: 1182-1194

Guo J, Peng Y, Guo J, Ma J, Wang W, Wang B. 2011. Dissolved organic matter in biologically treated sewage effluent (BTSE): Characteristics and comparison. Desalination, 278: 365-372

Guo W, Yang F, Li Y, Wang S. 2017. New insights into the source of decadal increase in chemical oxygen demand associated with dissolved organic carbon in Dianchi Lake. Sci Total Environ, 603: 699-708

Gupta D, Singh S K. 2012. Greenhouse gas emissions from wastewater treatment plants: A case study of Noida. J Water Sustainability, 2: 131-139

Hanaki K, Hong Z, Matsuo T. 1992. Production of nitrous oxide gas during denitrification of wastewater. Water Sci Technol, 26: 10271036

Hansell D A. 2013. Recalcitrant dissolved organic carbon fractions. Annu Rev Mar Sci, 5: 421-445

Hansen J E, Lacis A A. 1990. Sun and dust versus greenhouse gases:
An assessment of their relative roles in global climate change. Nature, 346: 713-719

Hao X, Liu R, Huang X. 2015. Evaluation of the potential for operating carbon neutral WWTPs in China. Water Res, 87: 424-431

Hudson N, Baker A, Ward D, Reynolds D M, Brunsdon C, CarliellMarquet C, Browning S. 2008. Can fluorescence spectrometry be used as a surrogate for the Biochemical Oxygen Demand (BOD) test in water quality assessment? An example from South West England. Sci Total Environ, 391: 149-158

Huo J, Jiang Y, Seaver W L, Robinson R B, Cox C D. 2006. Statistically based design of wastewater treatment plants (WWTPs) using Monte Carlo simulation of Activated Sludge Model No. 1 (ASM1). World Environmental \& Water Resources Congress

Ince B K, Ince O, Sallis P J, Anderson G K. 2000. Inert COD production in a membrane anaerobic reactor treating brewery wastewater. Water Res, 34: 3943-3948

IPCC. 2006. IPCC guidelines for national greenhouse gas inventories, Prepared by the National Greenhouse Gas Inventories Programme. In: Egglesto H S, Buendia L, Miwa K, Ngara T, Tanabe K, eds. Published, IGES, Japan

IPCC. 2014. Climate change 2014: Synthesis report. In: Pachauri R K, Meyer L A, eds. Contribution of Working Groups I, II and III to the Fifth Assessment Report of the Intergovernmental Panel on Climate Change. IPCC, Geneva. 151

Jiao N, Herndl G J, Hansell D A, Benner R, Kattner G, Wilhelm S W, Kirchman D L, Weinbauer M G, Luo T, Chen F, Azam F. 2010. Microbial production of recalcitrant dissolved organic matter: Longterm carbon storage in the global ocean. Nat Rev Microbiol, 8: 593599

Jiao N, Liu J, Edwards B, Lv Z, Cai R, Liu Y, Xiao X, Wang J, Jiao F, Wang R, Huang X, Guo B, Sun J, Zhang R, Zhang Y, Tang K, Zheng Q, Azam F, Batt J, Cai W J, He C, Herndl G J, Hill P, Hutchins D, LaRoche J, Lewis M, MacIntyre H, Polimene L, Robinson C, Shi Q, Suttle C A, Thomas H, Wallace D, Legendre L. 2021. Correcting a major error in assessing organic carbon pollution in natural waters. Sci Adv, 7: eabc7318

Jin G, Bao G, Feng S, Fang F. 2011. Effluent organic matter removal during advanced wastewater treatment process: O3-BAC and $\mathrm{AC} /$ O3-BAC. Second International Conference on Mechanic Automation \& Control Engineering

Jouanneau S, Recoules L, Durand M J, Boukabache A, Picot V, Primault Y, Lakel A, Sengelin M, Barillon B, Thouand G. 2014. Methods for assessing biochemical oxygen demand (BOD): A review. Water Res, 49: 62-82

Kallas J, Munter R. 1994. Post treatment of pulp and paper industry wastewater's using oxidation and adsorption processes. Water Sci 
Technol, 29: 259-272

Kappeler J, Gujer W. 1992. Estimation of kinetic parameters of heterotrophic biomass under aerobic conditions and characterization of wastewater for activated sludge modelling. Water Sci Technol, 25: $125-139$

Koutsou O P, Gatidou G, Stasinakis A S. 2018. Domestic wastewater management in Greece: Greenhouse gas emissions estimation at country scale. J Cleaner Prod, 188: 851-859

Kylefors K, Ecke H, Lagerkvist A. 2003. Accuracy of COD test for landfill leachates. Water Air Soil Pollution, 146: 153-169

Lashof D A, Ahuja D R. 1990. Relative contributions of greenhouse gas emissions to global warming. Nature, 344: 529-531

Lemaire R, Meyer R, Taske A, Crocetti G R, Keller J, Yuan Z. 2006. Identifying causes for $\mathrm{N}_{2} \mathrm{O}$ accumulation in a lab-scale sequencing batch reactor performing simultaneous nitrification, denitrification and phosphorus removal. J Biotechnol, 122: 62-72

Liu Y, Cheng X, Lun X, Sun D. 2014. $\mathrm{CH}_{4}$ emission and conversion from $\mathrm{A}^{2} \mathrm{O}$ and $\mathrm{SBR}$ processes in full-scale wastewater treatment plants. J Environ Sci, 26: 226-232

Lu D, Xiao K, Chen Y, Soh Y N A, Zhou Y. 2018. Transformation of dissolved organic matters produced from alkaline-ultrasonic sludge pretreatment in anaerobic digestion: From macro to micro. Water Res, 142: 138-146

Makinia J, Swinarski M, Dobiegala E. 2002. Experiences with computer simulation at two large wastewater treatment plants in northern Poland. Water Sci Technol, 45: 209-218

Makwana A R, Ahammed M M. 2017. Electrocoagulation process for the post-treatment of anaerobically treated urban wastewater. Separation Sci Tech, 52: 1412-1422

Mitsch W J, Bernal B, Nahlik A M, Mander Ü, Zhang L, Anderson C J, Jørgensen S E, Brix H. 2013. Wetlands, carbon, and climate change. Landscape Ecol, 28: 583-597

Mittal A. 2011. Biological wastewater treatment. Water Today, 1: 32-44 Mo W, Zhang Q. 2012. Can municipal wastewater treatment systems be carbon neutral? J Environ Manage, 112: 360-367

Montzka S A, Dlugokencky E J, Butler J H. 2011. Non- $\mathrm{CO}_{2}$ greenhouse gases and climate change. Nature, 476: 43-50

Nguyen T K L, Ngo H H, Guo W, Chang S W, Nguyen D D, Nghiem L D, Liu Y, Ni B, Hai F I. 2019. Insight into greenhouse gases emissions from the two popular treatment technologies in municipal wastewater treatment processes. Sci Total Environ, 671: 1302-1313

Poulsen T G, Hansen J A. 2009. Assessing the impacts of changes in treatment technology on energy and greenhouse gas balances for organic waste and wastewater treatment using historical data. Waste Manage Res, 27: 861-870

Ra C, Lo K, Shin J, Oh J, Hong B. 2000. Biological nutrient removal with an internal organic carbon source in piggery wastewater treatment. Water Res, 34: 965-973

Ragnauth S A, Creason J, Alsalam J, Ohrel S, Petrusa J E, Beach R H. 2015. Global mitigation of non- $\mathrm{CO}_{2}$ greenhouse gases: Marginal abatement costs curves and abatement potential through 2030. J Integrative Environ Sci, 12: 155-168

Räike A, Kortelainen P, Mattsson T, Thomas D N. 2012. 36 year trends in dissolved organic carbon export from Finnish rivers to the Baltic Sea. Sci Total Environ, 435-436: 188-201

Reynolds D M. 2002. The differentiation of biodegradable and nonbiodegradable dissolved organic matter in wastewaters using fluorescence spectroscopy. J Chem Technol Biotechnol, 77: 965972

Rodriguez-Garcia G, Hospido A, Bagley D M, Moreira M T, Feijoo G. 2012. A methodology to estimate greenhouse gases emissions in Life Cycle Inventories of wastewater treatment plants. Environ Impact Assessment Rev, 37: 37-46

de Souza S M A G U, Bonilla K A S, de Souza A A U. 2010. Removal of $\mathrm{COD}$ and color from hydrolyzed textile azo dye by combined ozonation and biological treatment. J Hazard Mater, 179: 35-42

Samudro G, Mangkoedihardjo S. 2010. Review on BOD, COD and BOD/COD ratio: A triangle zone for toxic, biodegradable and stable levels. Int J Acad Res, 2: 235-239

Sepp M, Koiv T, Noges P, Noges T. 2018. Do organic matter metrics included in lake surveillance monitoring in Europe provide a broad picture of brownification and enrichment with oxygen consuming substances? Sci Total Environ, 610-611: 1288-1297

Shannon M A, Bohn P W, Elimelech M, Georgiadis J G, Mariñas B J, Mayes A M. 2008. Science and technology for water purification in the coming decades. Nature, 452: 301-310

Union E, Parliament E. 2013. Regulation (EU) Nr. 575/2013 of the European Parliament and of the Council

Verma R, Suthar S. 2018. Performance assessment of horizontal and vertical surface flow constructed wetland system in wastewater treatment using multivariate principal component analysis. Ecol Eng, 116: 121-126

Wang J, Zhang J, Xie H, Qi P, Ren Y, Hu Z. 2011. Methane emissions from a full-scale $\mathrm{A} / \mathrm{A} / \mathrm{O}$ wastewater treatment plant. Bioresource Tech, 102: 5479-5485

Wang Z, Xu X, Gong Z, Yang F. 2012. Removal of COD, phenols and ammonium from Lurgi coal gasification wastewater using $\mathrm{A}^{2} \mathrm{O}$ MBR system. J Hazard Mater, 235: 78-84

Ward A J, Hobbs P J, Holliman P J, Jones D L. 2008. Optimisation of the anaerobic digestion of agricultural resources. Bioresource Tech, 99: 7928-7940

Wu J, Zhang J, Jia W, Xie H, Gu R R, Li C, Gao B. 2009. Impact of 
$\mathrm{COD} / \mathrm{N}$ ratio on nitrous oxide emission from microcosm wetlands and their performance in removing nitrogen from wastewater. Bioresource Tech, 100: 2910-2917

Wu L, Ning D, Zhang B, Li Y, Zhang P, Shan X, Zhang Q, Brown M R, Li Z, Van Nostrand J D, Ling F, Xiao N, Zhang Y, Vierheilig J, Wells G F, Yang Y, Deng Y, Tu Q, Wang A, Zhang T, He Z, Keller J, Nielsen P H, Alvarez P J J, Criddle C S, Wagner M, Tiedje J M, He Q, Curtis T P, Stahl D A, Alvarez-Cohen L, Rittmann B E, Wen X, Zhou J. 2019. Global diversity and biogeography of bacterial communities in wastewater treatment plants. Nat Microbiol, 4: 1183-1195

Wunderlin P, Mohn J, Joss A, Emmenegger L, Siegrist H. 2012. Mechanisms of $\mathrm{N}_{2} \mathrm{O}$ production in biological wastewater treatment under nitrifying and denitrifying conditions. Water Res, 46: 1027-
1037

Yan N, Ck A, Rdw C, Dcsa D. 2020. Composition and biotransformational changes in soluble microbial products (SMPs) along an anaerobic baffled reactor (ABR). Chemosphere, 254: 126775

Yu X, Xu R, Wei C, Wu H. 2016. Removal of cyanide compounds from coking wastewater by ferrous sulfate: Improvement of biodegradability. J Hazard Mater, 302: 468-474

Zhang B, Ning D, Yang Y, Van Nostrand J D, Zhou J, Wen X. 2020. Biodegradability of wastewater determines microbial assembly mechanisms in full-scale wastewater treatment plants. Water Res, 169: 115276

Ziemiński K, Frąc M. 2012. Methane fermentation process as anaerobic digestion of biomass: Transformations, stages and microorganisms. Afr J Biotechnol, 11: 4127-4139

(责任编委: 翦知淐) 